Received: 17 February 2017

Accepted: 10 July 2017

Published online: 08 August 2017

\section{Wheat TaPUB1 modulates plant drought stress resistance by improving antioxidant capability}

\author{
Guangqiang Zhang ${ }^{1}$, Meng Zhang ${ }^{1,2}$, Zhongxian Zhao ${ }^{1}$, Yuanqing Ren ${ }^{1}$, Qinxue $\mathrm{Li}^{1}$ \& \\ Wei Wang ${ }^{1}$
}

E3 ligases play significant roles in plant stress tolerance by targeting specific substrate proteins for post-translational modification. In a previous study, we cloned TaPUB1 from Triticum aestivum L., which encodes a U-box E3 ligase. Real-time polymerase chain reaction revealed that the gene was up-regulated under drought stress. To investigate the function of TaPUB1 in the response of plants to drought, we generated transgenic Nicotiana benthamiana ( $N$. benthamiana) plants constitutively expressing TaPUB1 under the CaMV35S promoter. Compared to wild type (WT), the transgenic plants had higher germination and seedling survival rates as well as higher photosynthetic rate and water retention, suggesting that the overexpression of TaPUB1 enhanced the drought tolerance of the TaPUB1 overexpressing (OE) plants. Moreover, less accumulation of reactive oxygen species (ROS) and stronger antioxidant capacity were detected in the OE plants than in the WT plants. To characterize the mechanisms involved, methyl viologen (MV) was used to induce oxidative stress conditions and we identified the functions of this gene in the plant tolerance to oxidative stress. Our results suggest that TaPUB1 positively modulates plant drought stress resistance potential by improving their antioxidant capacity.

In the natural environment, plants are consistently exposed to a series of unfavorable conditions that affects their growth and development. Over the course of evolution, plants have acquired complex and comprehensive mechanisms to withstand these unfavorable stress conditions ${ }^{1}$; these include sophisticated physiological and biochemical regulatory networks ${ }^{2}$. The major mechanisms involved in adaptation to water stress include reduced water loss, enhanced water uptake and its efficient use, and production of low-molecular-weight osmolytes that sustain cellular functions under drought conditions. A number of antioxidants and enzymes scavenge the reactive oxygen species, such as superoxide and singlet oxygen, which are generated due to water deficit. At the molecular level, several drought-inducible genes and transcription factors function in establishing the stress tolerance network in plants ${ }^{3-5}$

The ubiquitin-26S proteasome system (UPS) is an extremely complex and versatile system for protein degradation that plays important roles in the response of all eukaryotic species to varied environmental conditions, such as those of drought and salt stress ${ }^{6,7}$. In recent years, data have shown that the UPS targets many intracellular regulators that play key roles in hormone signaling pathways, responses to abiotic stresses, and in battling pathogens ${ }^{8}$. The ubiquitination process involves the concerted action of an intricately linked network of enzymes, namely E1, E2, and E3. Of these three proteins, the E3 ubiquitin ligases are the most diverse proteins in the UPS that specifically recognize an extensive range of targets ${ }^{9}$. Depending on the type of substrate, E3 ubiquitin ligases can be subdivided into the following kinds: F-box type, RING type, U-box type, and HECT type ${ }^{10}$.

The U-box type of E3 ubiquitin ligase has a highly conserved domain of 70 amino acids that forms a scaffold structure. The plant U-box (PUB) proteins contain some distinct categories, implying that they have a wide array of roles. Lack or mutation of the U-box has been demonstrated to abolish the ubiquitination activity ${ }^{11,12}$. In recent years, it has been reported that some U-box proteins participate in the organism's response to hormones ${ }^{13,14}$, and tolerance to biotic ${ }^{15,16}$ and abiotic stresses ${ }^{17,18}$.

${ }^{1}$ State Key Laboratory of Crop Biology, Shandong Key Laboratory of Crop Biology, College of Life Sciences, Shandong Agricultural University, Tai'an, Shandong, 271018, P.R. China. ${ }^{2}$ Collaborative Innovation Center, Jining Medical University, Jining, Shandong, 272067, P.R. China. Correspondence and requests for materials should be addressed to W.W. (email: wangw@sdau.edu.cn) 


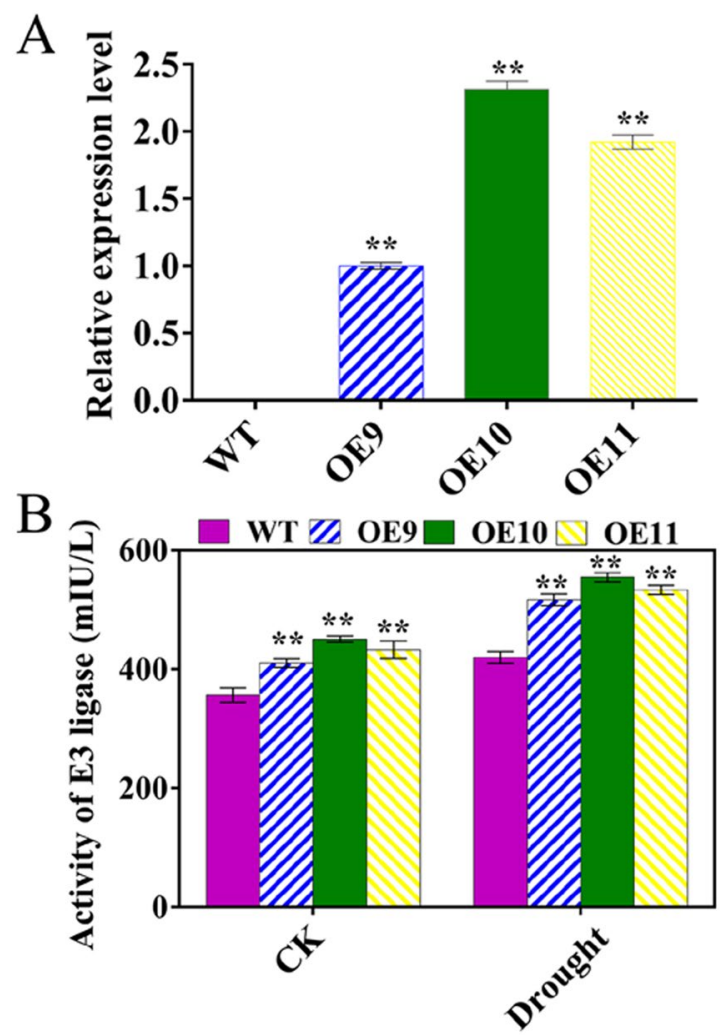

Figure 1. Confirmation of TaPUB1 expression in transgenic $N$. benthamiana lines. (A) The relative expression levels of TaPUB1 in the WT plants and three different TaPUB1 transgenic lines (OE9, OE10, and OE11). (B) The E3 ligase activity in leaves of OE and WT plants. The experiments were repeated three times and the bars indicate SEs. * and ** indicate significant differences at $\mathrm{P}<0.05$ and $\mathrm{P}<0.01$ in the values among the three overexpression lines and the WT plants.

Wheat is the third largest crop in the world and is consumed by more than $35 \%$ of the world population. The main constraints in wheat cultivation are the scarcity of planting area and water. Therefore, studies on drought tolerance and resistance are very important for wheat production ${ }^{19,20}$. In a previous study, we cloned a U-box protein gene from wheat and named it TaPUB1. Overexpression of TaPUB1 could confer enhanced salt tolerance in transgenic N. benthamiana. Moreover, drought stress could induce the expression of TaPUB1. In this study, we present and discuss the results of our investigation on the physiological and molecular mechanisms of increased drought tolerance upon TaPUB1 overexpression.

\section{Results}

Selection of 35S::TaPUB1 transgenic N. benthamiana plants and detection of E3 ligase activity. In our previous study, the full-length cDNA of TaPUB1 (GenBank ID: JX307854) was obtained from Triticum aestivum L. 'HF9703'. In this research, we chose plants from three 35S::TaPUB1 transgenic N. benthamiana lines (OE9, OE10, OE11) and WT to study the resistance of the OE plants to drought. The TaPUB1 transgenic plants were authenticated by qRT-PCR. All the three independent homozygous lines showed different levels of expression of TaPUB1 whereas no expression was observed in WT (Fig. 1A). We also measured the E3 ligase activity and its response to drought stress in the leaves of OE and WT lines. Drought stress was observed to increase E3 ligase activity in all the plants; the activity was higher in the OE plants than in the WT under both normal and drought conditions (Fig. 1B). These results provided evidence that TaPUB1 had stable expression in the N. benthamiana plants and increased the activity of E3 ligase substantially.

Reduced sensitivity of germination of TaPUB1 overexpressing $N$. benthamiana seeds to drought stress. The expression of TaPUB1 was upregulated following PEG6000 treatment. Thereafter, the function of TaPUB1 in response to drought stress was investigated using the three TaPUB1-overexpressing lines. Firstly, the percentage of seed germination in the transgenic and WT plants under water stress conditions was determined. The seeds of WT plant and the three TaPUB1-overexpressing lines were germinated on petri dishes containing water or PEG6000 (Fig. 2A). In the absence of PEG6000, the germination of seeds from the OE lines was comparable with those from the WT plants. In the presence of 5\% PEG6000, we determined slight difference in germination rates between WT and OE seeds. However, the germination percentage in the case of transgenic lines was distinctly higher than that in the WT plants on the medium containing 10\% PEG6000 (Fig. 2A,B). These results provide evidence that during the seed germination stage, the transgenic plants were less sensitive to drought stress than were the WT plants. 
A

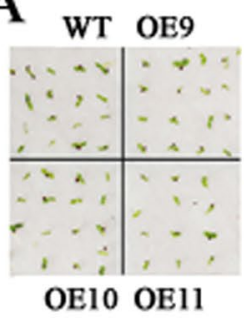

CK

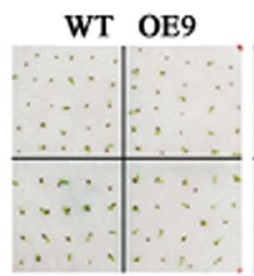

OE10 OE11

$5 \%$ PEG6000

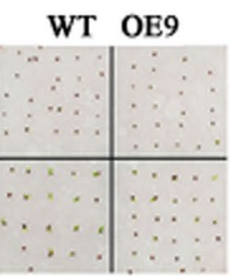

OE10 OE11

$10 \%$ PEG6000

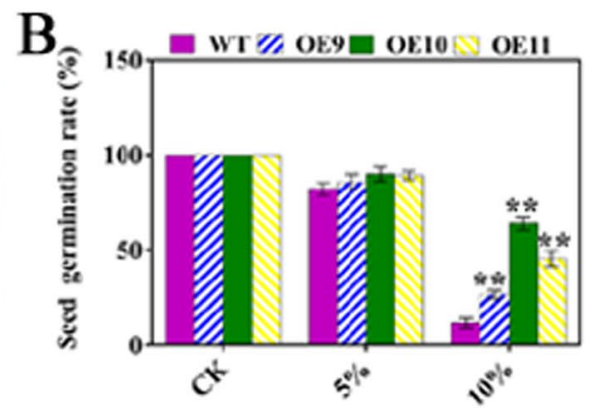

C

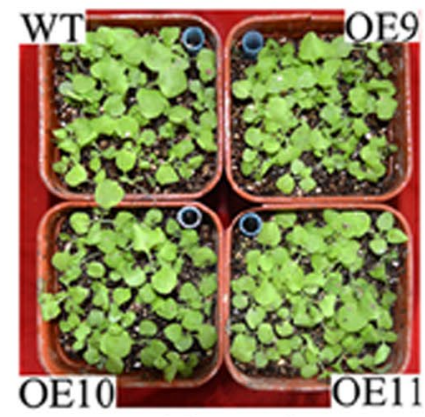

Normal

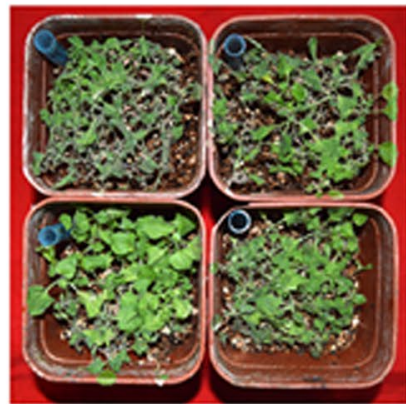

Treatment of drought

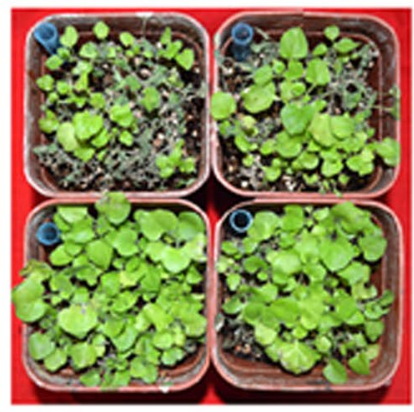

Rewater
D

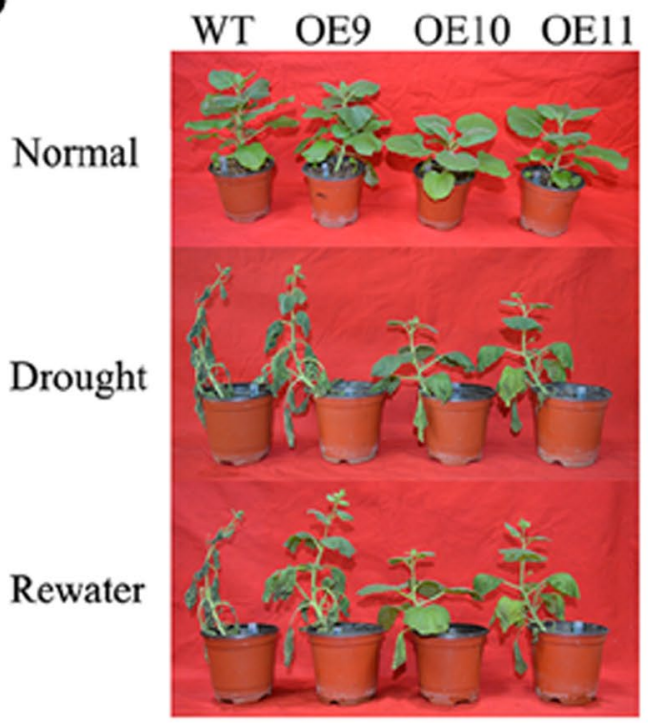

$\mathrm{E}$

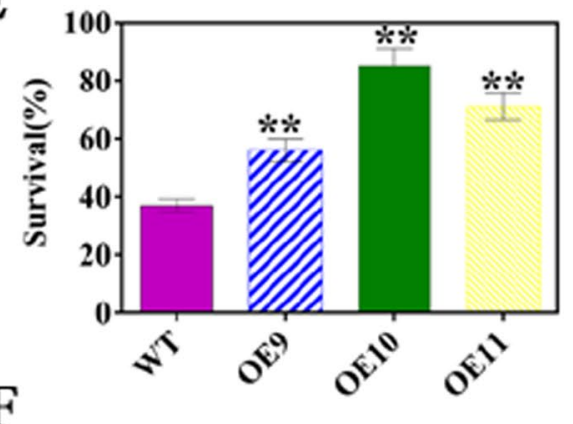

$\mathrm{F}$

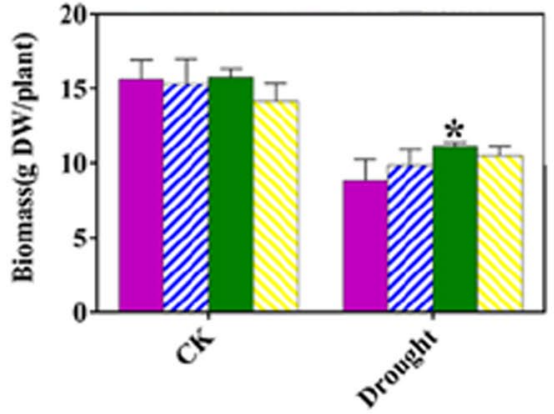

Figure 2. Influence of drought stress on the growth of TaPUB1-overexpressing and WT plants. (A) Phenotype of seedlings grown on MS medium containing different concentrations of PEG6000 for $7 \mathrm{~d}$. (B) Comparison of the germination rates of TaPUB1-overexpressing and WT plants. Values are means \pm SEs of at least three independent experiments ( $\mathrm{n}=45$ seeds per treatment). (C) The phenotypes of 2 -week-old TaPUB1overexpressing and WT plants subjected to drought treatment for $5 \mathrm{~d}$ and to subsequent re-watering for $2 \mathrm{~d}$. (D) The survival ratio was calculated as the percentage of plants that resumed growth $2 \mathrm{~d}$ after re-watering. (E) Phenotypes of OE and WT plants exposed to water withholding treatment at the 2-month-old stage. Representative plants subjected to drought stress for $5 \mathrm{~d}$. After $5 \mathrm{~d}$ of drought treatment, the plants were rewatered and their recovery was recorded. (F) The biomass of TaPUB1-overexpressing and WT plants. The experiments were repeated three times and the bars indicate SEs. * and ** indicate significant differences at $\mathrm{P}<0.05$ and $\mathrm{P}<0.01$ in the values among the three overexpression lines and the WT plants. 
A

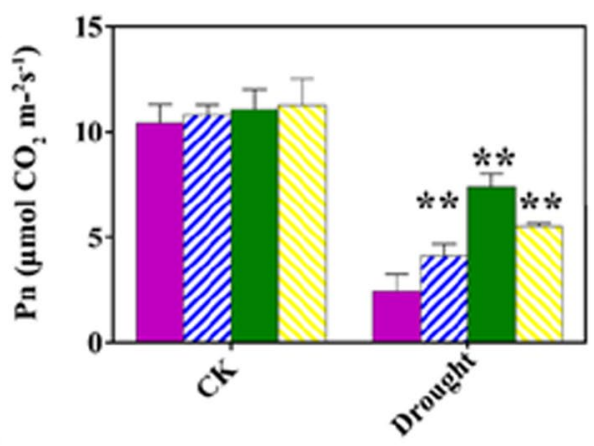

$\mathrm{C}$

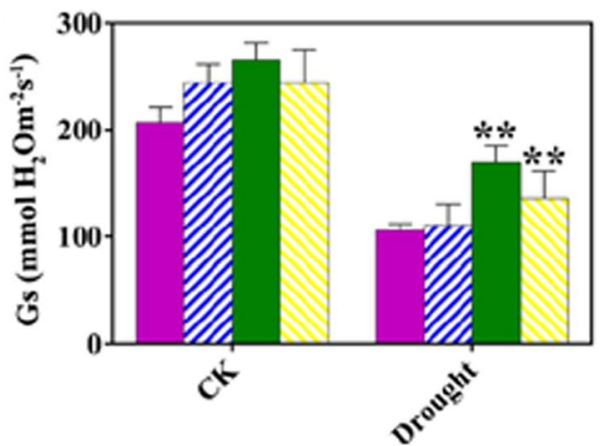

$\mathrm{E}$

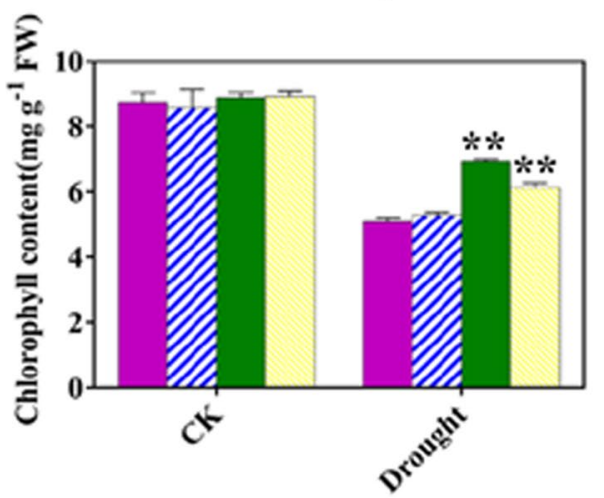

$\mathrm{B}$

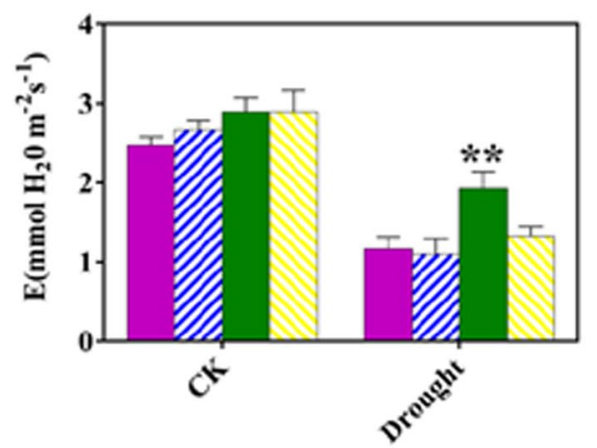

$\mathrm{D}$

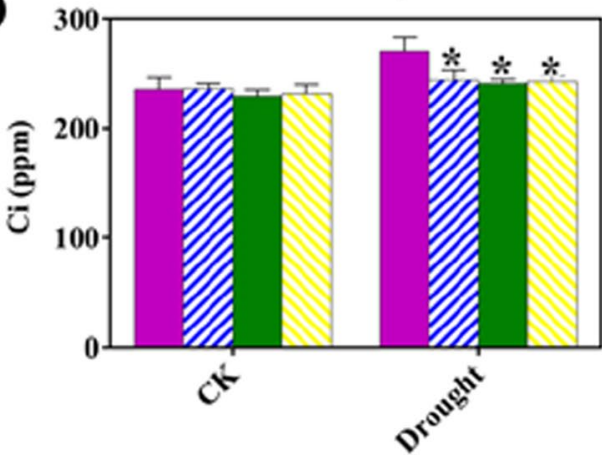

WT $\square$ OE10

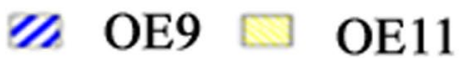

Figure 3. Photosynthetic performance of TaPUB1-overexpressing lines and WT plants after drought treatment. (A) Net photosynthetic rate, Pn. (B) Transpiration rate, E. (C) Stomatal conductance, Gs. (D) Intercellular $\mathrm{CO}_{2}$ concentration, Ci. (E) Chlorophyll content. The experiments were repeated three times and the bars indicate SEs. ${ }^{*}$ and $* *$ indicate significant differences at $\mathrm{P}<0.05$ and $\mathrm{P}<0.01$ among the three overexpression lines and the WT plants.

Overexpression of TaPUB1 enhanced the drought tolerance of transgenic seedlings and adult $\boldsymbol{N}$. benthamiana plants. The OE and WT seeds were sown in roseite and after two weeks of growth of the seedlings, watering was withheld for $5 \mathrm{~d}$. After this drought stress, the seedlings were rewatered for $2 \mathrm{~d}$. The survival rates of transgenic plants exposed to this treatment ranged between $56 \%$ and $81 \%$ whereas that of WT was 37\% (Fig. 2C,E).

Furthermore, two-month-old $N$. benthamiana plants were allowed to dehydrate naturally for $5 \mathrm{~d}$, and were subsequently rewatered for $3 \mathrm{~d}$. After this drought treatment, the TaPUB1-overexpressing plants revealed more robust phenotypes, including higher biomass, than the WT plants (Fig. 2D,F). To further validate the drought tolerance of OE plants, two-month-old transgenic lines and WT plants were subjected to drought stress for $5 \mathrm{~d}$, and the photosynthetic parameters of the plants were examined using CIRAS-2 (Hitchin, UK) photosynthesis system. The results showed that drought stress resulted in a significant decline in the net photosynthetic rate (Pn), transpiration rate (E), and stomatal conductance (Gs) in both the OE and WT plants (Fig. 3A-C). However, the Pn in the OE plants was higher than that in the WT plants (Fig. 3A) under drought treatment. Similar results were observed for E (Fig. 3B) and Gs (Fig. 3C). The chlorophyll content of the OE lines was also higher than that of the WT plants under stress conditions (Fig. 3E). However, the intercellular $\mathrm{CO}_{2}$ concentration (Ci) was lower in the transgenic plants than it was in the WT plants (Fig. 3D). Overall, the results depicted in Figs 2, 3 and 4 indicate that overexpression of TaPUB1 increased the resistance to drought in plants. 

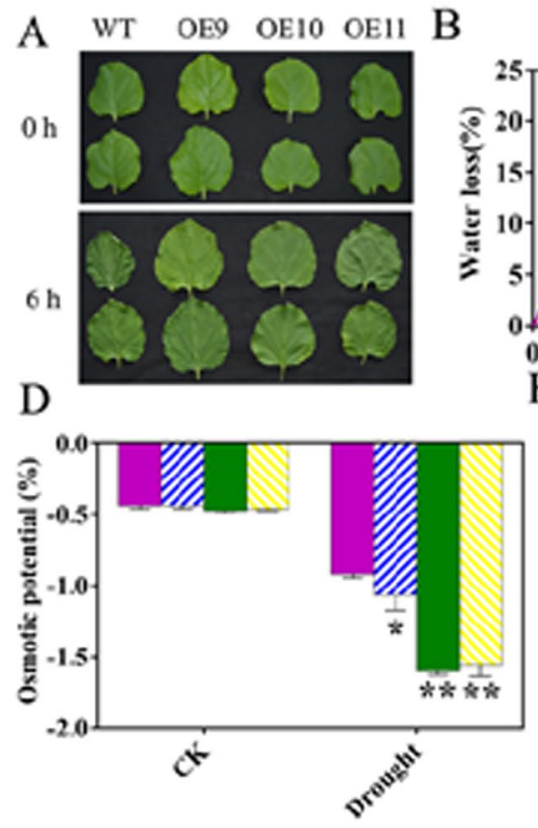

B

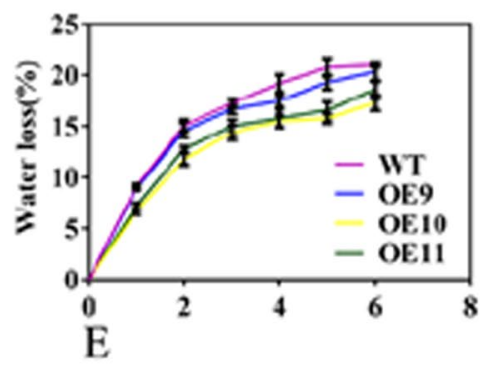

$\mathrm{C}$

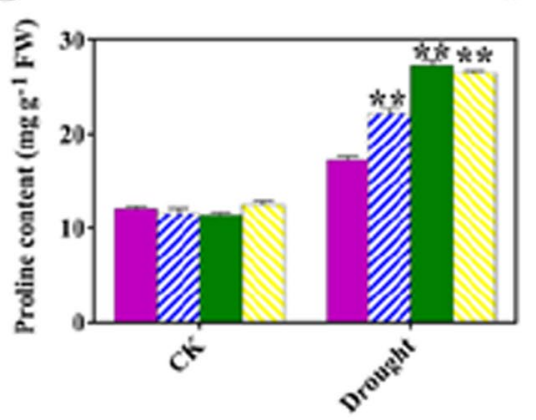

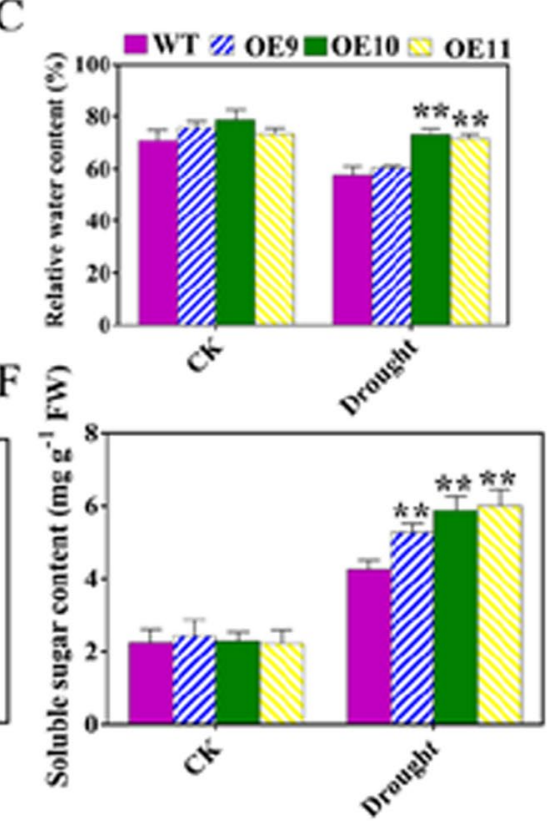

Figure 4. Influence of drought stress on water loss, relative water content, osmotic potential, proline content, and soluble sugar content in the OE and WT plants. The phenotypes and kinetics of water loss from the leaves of 2-month-old N. benthamiana lines (A,B). (C) Relative water content. Blades separated from 2-month-old TaPUB1-overexpressing and WT lines after drought treatment. (D) Osmotic potential. (E) Proline content and (F) Soluble sugar content after drought treatment. The experiments were repeated three times and the bars indicate SEs. * and ** indicate significant differences at $\mathrm{P}<0.05$ and $\mathrm{P}<0.01$ among the three overexpression lines and the WT plants.

Overexpression of TaPUB1 increased the water retention capacity of OE lines. To determine the water retention capacity of transgenic $N$. benthamiana plants, we measured the rate of water loss, relative water content (RWC), osmotic potential, and the accumulation of compatible solutes, including proline and soluble sugars. The detached leaves of OE plants were observed to have lost less water than those of WT plants within $6 \mathrm{~h}$ of dehydration (Fig. 4A,B). There were no significant differences in the leaf RWC among the WT and OE lines under water-replete condition. In contrast, after drought the RWC in OE plants was higher than that in the WT plants (Fig. 4C). Moreover, the osmotic potential of the transgenic plants was lower than that of the WT plants (Fig. 4D) after the drought treatment. At the same time, the contents of proline and soluble sugars were higher in the OE plants than in the WT plants (Fig. 4E,F). This suggests that the water retention ability of the transgenic lines was better than that of the WT plants.

Influence of drought on electrolyte leakage, MDA content, and protein carbonylation in the TaPUB1-overexpressing plants. When a plant tissue is subject to stresses, the membrane structure of its cells is impaired, which leads to the increase in the membrane permeability and the electrolyte leakage is also increased, consequently. The electrolyte leakage levels in the OE and WT plants were similar in the absence of drought stress. However, the transgenic plants maintained lower levels of electrolyte leakage that those in the WT plants after the drought treatment (Fig. 5A). The MDA content of the OE and WT plants increased after the drought treatment, but the increase was less in the OE plants than that in the WT plants (Fig. 5B). These results indicate that the membrane injury in the transgenic plants was less than that in the WT plants.

All the amino acids are sensitive to oxidative stress. The protein carbonyl content increases significantly after oxidative modification. The protein carbonyl content can be used as an indicator of protein oxidative damage ${ }^{21}$. The protein carbonyl levels of the transgenic and WT plants were detected by immunoblotting. Although the protein carbonyl content increased in both the transgenic and the WT plants after drought treatment (Fig. 5C,D), the levels in the transgenic plants were observably lower than that in the WT plants. Overall, these results showed that the damage in the OE plants was less than that in the control plants after the drought treatment (Fig. 5).

Overexpression of TaPUB1 reduced the accumulation of ROS in the transgenic $N$. benthamiana plants under drought stress. The production and accumulation of ROS can be induced by drought treatment. We examined the levels of endogenous hydrogen peroxide $\left(\mathrm{H}_{2} \mathrm{O}_{2}\right)$ by $3,3^{\prime}$-diaminobenzidine (DAB) staining and those of superoxide radical $\left(\mathrm{O}_{2}^{--}\right)$by nitrotetrazolium blue chloride (NBT) staining. After imposition of drought stress, different levels of $\mathrm{H}_{2} \mathrm{O}_{2}$ were detected in the seedlings of the WT and transgenic lines; however, the formation of brown precipitate in WT plants was much more than in the transgenic lines (Fig. 6A). Similar results were observed for $\mathrm{O}_{2}^{--}$production (Fig. 6B). The quantitative results of $\mathrm{H}_{2} \mathrm{O}_{2}$ content and $\mathrm{O}_{2}^{--}$production rate 
A

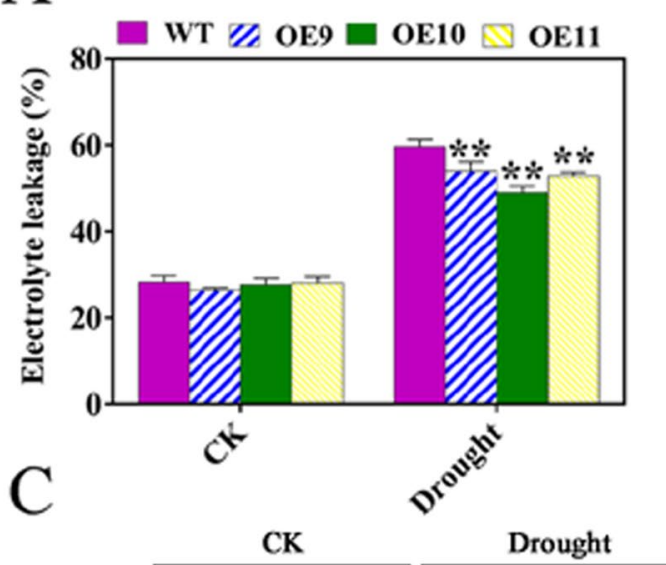

Marker WT OE9 OE10 OE11WT OE9 OE10 OE11

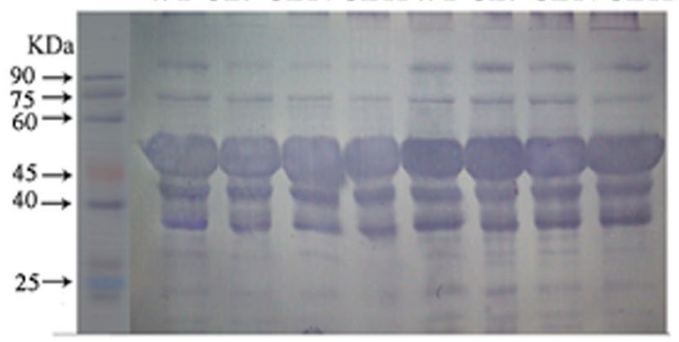

$\mathrm{B}$
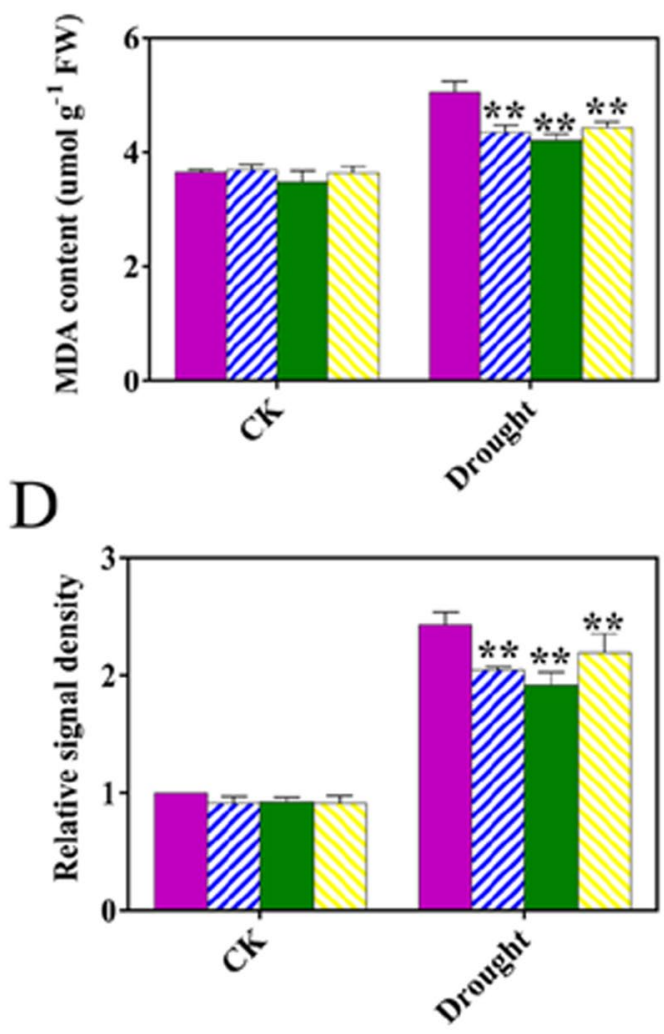

Figure 5. Influence of drought stress on electrolyte leakage, malondialdehyde (MDA), and protein carbonylation levels in the OE and WT plants. (A) Electrolyte leakage. (B) MDA content. (C) Protein carbonylation levels. (D) Relative signal density of protein carbonylation levels observed in (C). The experiments were repeated three times and the bars indicate SEs. * and ** indicate significant differences at $\mathrm{P}<0.05$ and $\mathrm{P}<0.01$ among the three overexpression lines and the WT plants.

were consistent with the results of staining (Fig. 6C,D). These results suggested that the overexpression of TaPUB1 reduced the accumulation of ROS in the OE lines.

Antioxidant enzymes can effectively remove ROS. The UPS is also involved in clearance of oxidized proteins from cells ${ }^{22}$. We evaluated the activity of four antioxidant enzymes, including superoxide dismutase (SOD), peroxidase (POD), catalase (CAT), and ascorbate peroxidase (APX) in the transgenic and WT plants (Fig. S1). There were no obvious differences in the activities of the four antioxidant enzymes mentioned above, among the plants under normal conditions. However, under drought stress, the activities of all the antioxidant enzymes in the transgenic plants were significantly higher than those in the WT plants.

TaPUB1 overexpression alters the expression of oxidation- and drought-related genes. To further elucidate the possible molecular mechanisms of the increased drought tolerance in the transgenic $N$. benthamiana plants, we performed qPCR analysis of a set of known antioxidant- and drought-related genes in $N$. benthamiana plants; the genes whose expression were assessed included dehydration-responsive element binding $(N b D R E B)$, early responsive to dehydration $(N b E R D)$, 9-cis-epoxycarotenoid dioxygenase (NbNCED), late embryogenesis-abundant protein ( $N b L E A)$, pyrroline-5-carboxylate synthase (NbP5CS), and antioxidant-related genes. including ascorbate peroxidase $(N b A P X)$, superoxide dismutase $(N b S O D)$, catalase $(N b C A T)$, glutathione $\mathrm{S}$-transferases ( $\mathrm{NbGST}$ ), and respiratory burst oxidase homolog A ( $\mathrm{NbRbohA}$ ). The expression of drought-related genes in the transgenic and WT plants showed varying degrees of upregulation after drought treatment (Fig. 7A-J). However, the increase in expression was higher in the OE plants than that in the WT plants. Therefore, the expression levels of all these stress-inducible marker genes were up-regulated by drought stress and by overexpression of TaPUB1 in N. benthamiana plants.

TaPUB1 overexpression improved the antioxidant capacity of transgenic $\boldsymbol{N}$. benthamiana lines. The results depicted in Figs 6 and S1 showed that the overexpression of TaPUB1 alleviated the accumulation of ROS and enhanced the antioxidant capacity of the transgenic plants under drought stress. Oxidative stress is a common secondary stress in almost all kinds of stress conditions ${ }^{23}$ and is also the primary mechanism by which biotic and abiotic stresses influence and regulate the biological metabolism ${ }^{24}$. To study the involvement of the antioxidant ability in the improved drought tolerance of the TaPUB1-overexpressing N. benthamiana plants, we simulated oxidative stress in plants by applying methyl viologen (MV). 
A

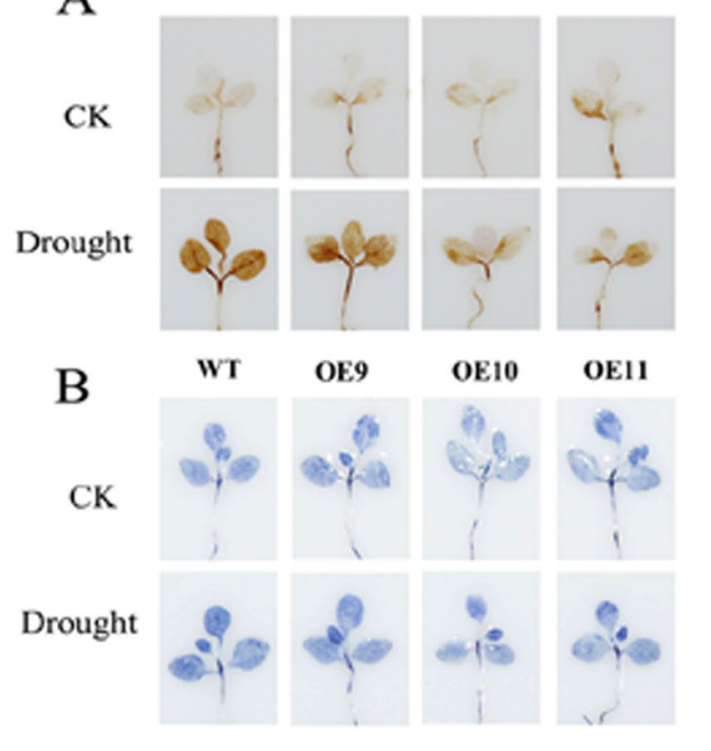

$\mathrm{C}$

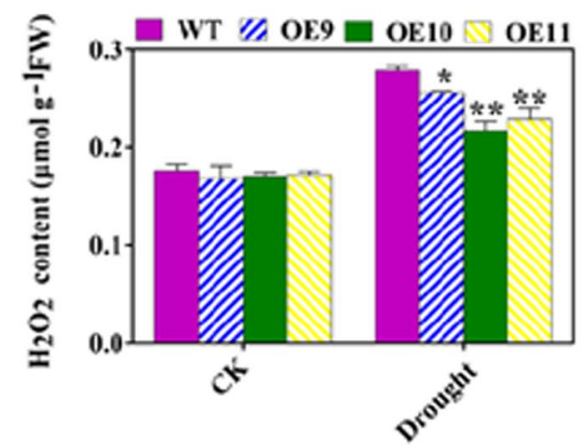

$\mathrm{D}$

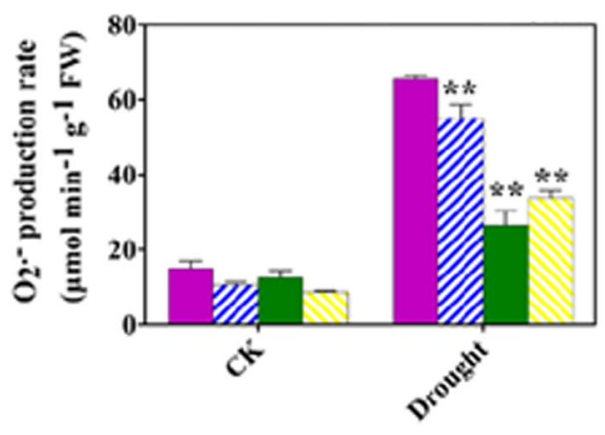

Figure 6. Influence of drought stress on ROS accumulation in the $\mathrm{OE}$ and WT plants. In situ detection of $\mathrm{H}_{2} \mathrm{O}_{2}$ (A) and $\mathrm{O}_{2}^{--}$(B) by DAB and NBT staining, respectively, in TaPUB1 overexpression and WT seedlings grown on roseite medium for two weeks and treated with $100 \mathrm{mM}$ mannitol, thereafter, for $7 \mathrm{~d}$. (C) $\mathrm{H}_{2} \mathrm{O}_{2}$ content and (D) $\mathrm{O}_{2}^{--}$production rate. The experiments were repeated three times and the bars indicate SEs. * and ** indicate significant differences at $\mathrm{P}<0.05$ and $\mathrm{P}<0.01$ among the three overexpression lines and the WT plants.

The transgenic and WT seeds were sown in MV-free or MV-containing Murashige and Skoog's (MS) culture medium. In the MV-free MS medium, the seeds of the TaPUB1-overexpressing lines showed similar germination rates to those of the WT plants. However, the number of seedlings with green cotyledons was higher in the transgenic plants than that in the WT on medium containing MV (Fig. 8A). The statistical analysis showed that the transgenic seeds had higher germination rate than the WT seeds (Fig. 8B).

We next assayed the growth responses of the OE seedlings to oxidative stress. Under normal conditions, the root morphology and length were similar in both the WT and transgenic lines, whereas in the presence of MV, the root was longer in the TaPUB1 overexpressing plants than that in the WT plants (Fig. 8C). The root of the TaPUB1 overexpressing lines was $18 \%$ and $34 \%$ longer than that of the WT plants in the presence of 5 and $10 \mu \mathrm{M}$ MV, respectively (Fig. 8D). Similar results were obtained for the fresh weight of seedlings (Fig. 8E).

We placed leaf discs from 2-month-old plants in Petri dishes containing different concentrations of MV. The exposure to different concentrations of MV for $3 \mathrm{~d}$ resulted in the manifestation of chlorosis in the leaf discs of the WT and OE plants; however, the leaf discs of the WT displayed much more chlorotic spots than those of the OE plants (Fig. 8F). This was consistent with the results of the chlorophyll content (Fig. 8G).

We further detected the accumulation of ROS in the leaves of the transgenic and WT plants after 12 and $24 \mathrm{~h}$ of MV treatment. The results showed that a considerably lower amount of ROS accumulated in the TaPUB1-overexpressing plants than in the WT plants (Fig. S2A,B). The quantitative results of the $\mathrm{H}_{2} \mathrm{O}_{2}$ content and $\mathrm{O}_{2}^{-}$production rates were similar to the results of staining (Fig. S2C,D). In addition, the activities of CAT and POD in the transgenic plants were significantly higher than those in the WT plants under oxidative stress (Fig. S2E,F).

The results described in Figs 8 and S2 suggest that TaPUB1 overexpression enhanced the oxidative stress tolerance of the OE plants.

\section{Discussion}

The PUB(U-box) proteins, as members of the E3 ligase family, have many important biological functions ${ }^{17,25,26}$. In a previous study, we obtained a U-box protein gene, TaPUB1, from wheat by homologous cloning. In order to find its functions in abiotic stress tolerance, we constructed the overexpression vector of TaPUB1 and generated heterologous overexpression N. benthamiana. We selected three independent homozygous transgenic T3 lines (OE9, OE10, OE11) with different TaPUB1 expression levels (Fig. 1A) for this study. Many U-box-containing proteins have been shown to have E3 ligase activity ${ }^{25}$. Sequence alignment and phylogenetic tree analysis indicated that TaPUB1 might encode a U-box protein. In the present study, the higher levels of E3 ligase activity were observed in the OE lines than that in the WT plants under both normal and drought conditions (Fig. 1B), suggesting the role of TaPUB1 in E3 activity.

The structures and functions of the PUB E3 family members have been identified and analyzed in several plants $^{27,28}$. Researches had shown that U-box proteins play important roles in the response of plants to abiotic 

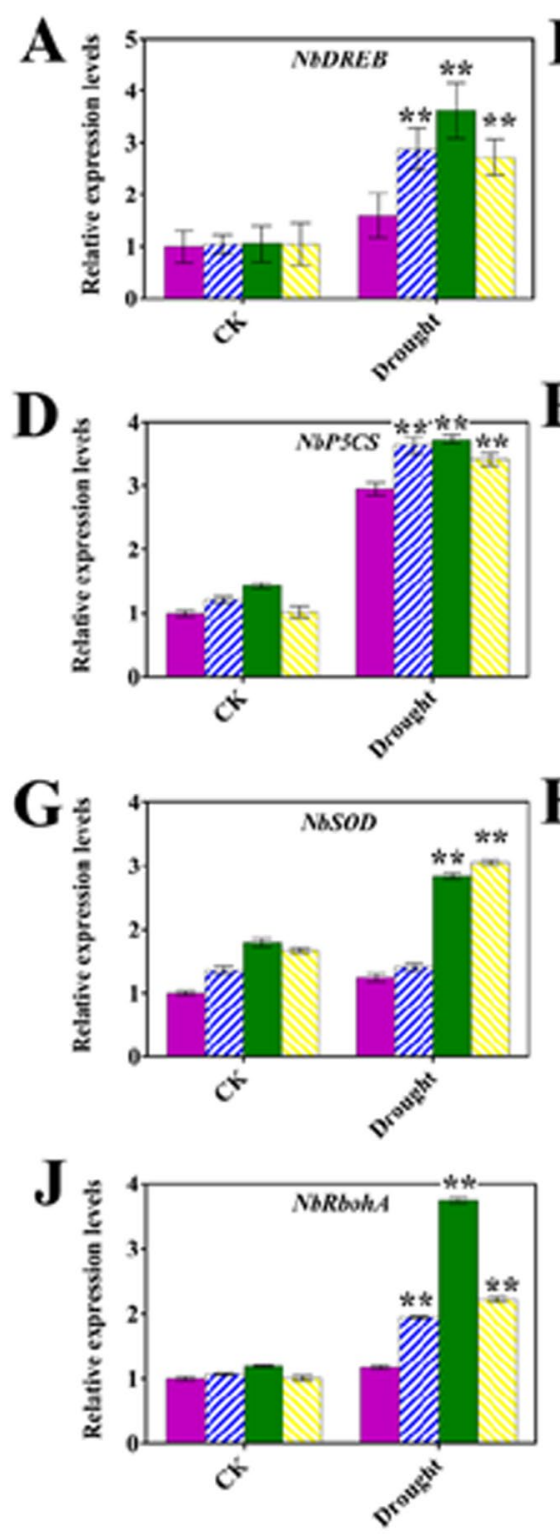

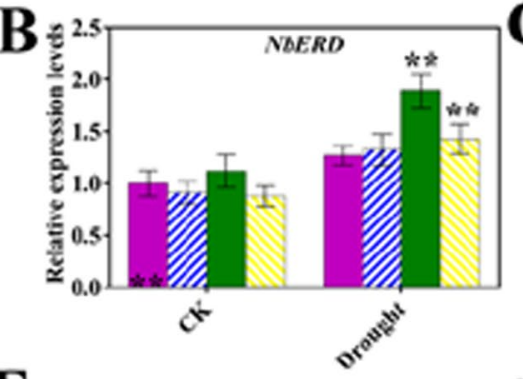

$\mathbf{E}$

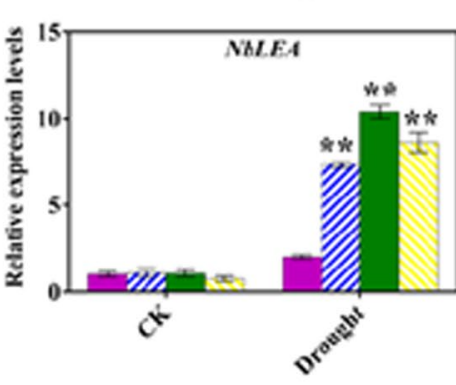

$\mathbf{H}$<smiles>[AlH2]</smiles>

I

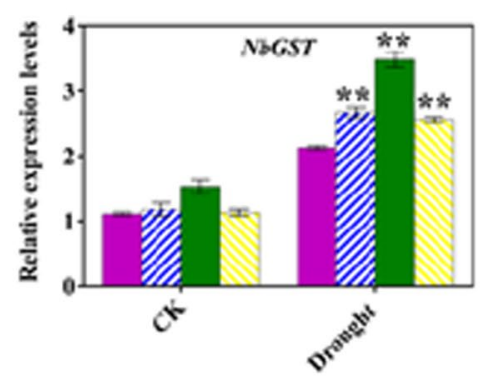

$\mathbf{F}$
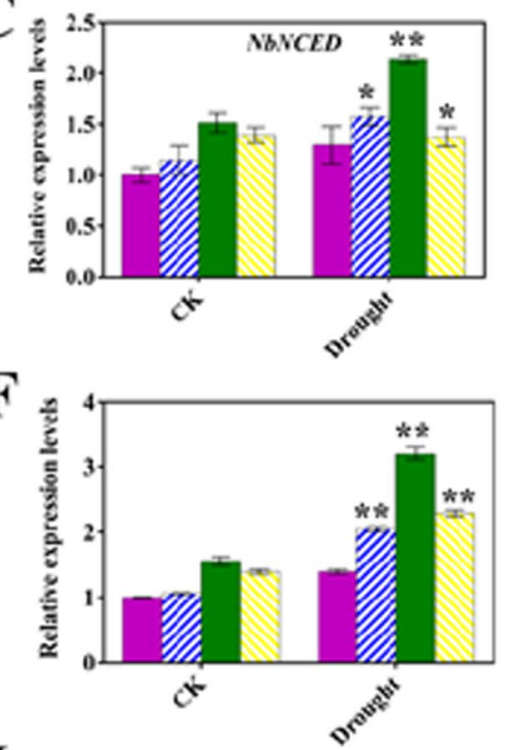

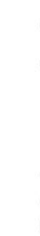

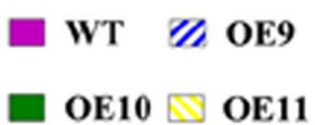

Figure 7. Expression of some antioxidant-and drought-related genes in OE and WT plants under drought stress. The histograms indicate the expression levels of the mentioned genes (A-J) in the transgenic and WT plants relative to their levels in the WT plants, which were considered as 1. Expression was normalized to the level of expression of actin in each sample was taken as the control. The experiments were repeated three times and the bars indicate SEs. * and ** indicate significant differences at $\mathrm{P}<0.05$ and $\mathrm{P}<0.01$ in the values among the three overexpression lines and the WT plants.

stresses $^{29}$. For instance, AtCHIP is involved in response to temperature stress in Arabidopsis ${ }^{30}$. OsPUB15 has an important effect in alleviating cellular oxidative stress ${ }^{31}$. Soybean E3 ubiquitin ligase, GmPUB8, is involved in negative regulation of drought response in Arabidopsis ${ }^{18}$. In this research, the transcript levels of TaPUB1 were also up-regulated by drought stress, suggesting that TaPUB1 may be involved in the plant drought stress response.

The effects of water stress range from morphological to molecular levels and are obvious at all phenological stages of plant growth whenever the drought takes place 5 . Seed germination is the first critical step in plant growth and development and water stress significantly delays the onset and reduces the rate of seed germination ${ }^{32,33}$. From Fig. 2, it is clear that the transgenic lines had significantly higher germination rates than the WT lines under PEG-induced water stress. Water shortage has a very large impact both on seedling and vegetative stages. The improved survival rates, more robust phenotypes, including more green leaves and higher biomass of transgenic plants were observed under drought stress (Fig. 2). These results indicate that TaPUB1 plays a positive role in plant drought tolerance.

Photosynthesis is one of the key processes affected by water deficit because of the decreased $\mathrm{CO}_{2}$ diffusion to the chloroplast and metabolic constraints ${ }^{34,35}$. Our data showed that the OE lines maintained higher Pn and chlorophyll contents than did the WT plants after the drought treatment (Fig. 3A,C,E). Although the Gs (Fig. 3C) 


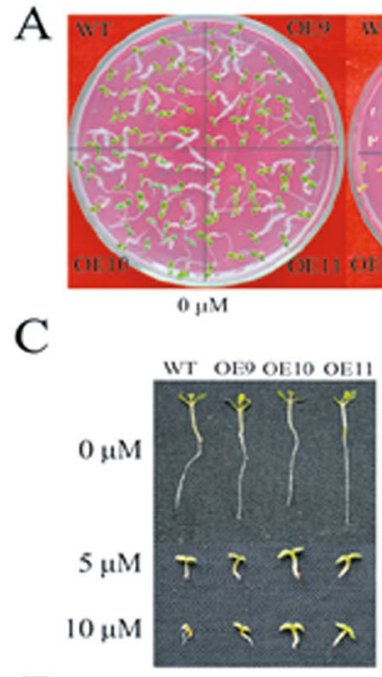

$\mathrm{F}$

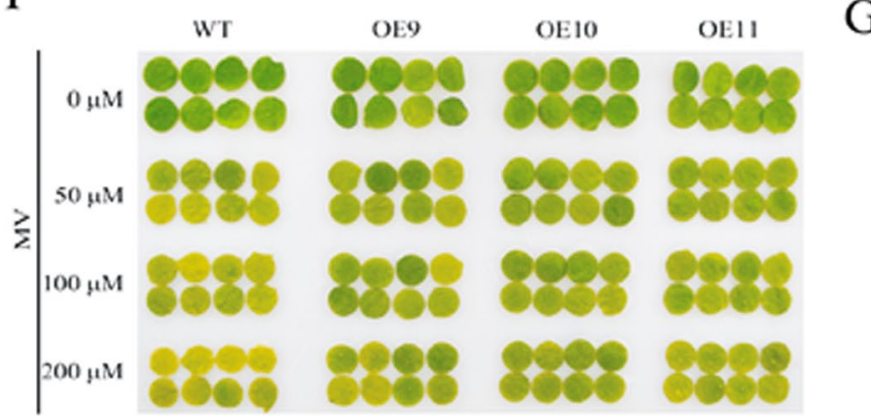

$\mathrm{D}$
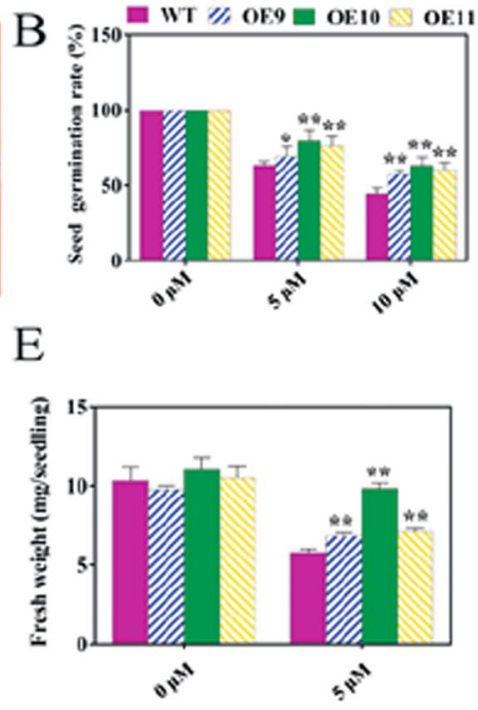

$\mathrm{G}$

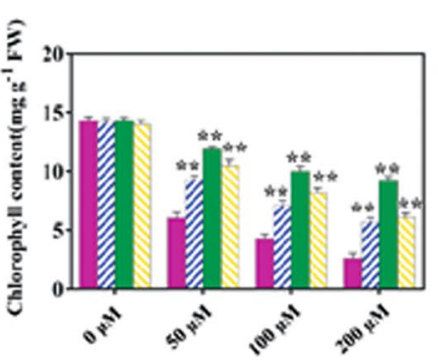

Figure 8. Analysis of oxidative stress tolerance in TaPUB1-overexpressing N. benthamiana plants. (A) Germination of seeds on MV-free or MV-containing MS culture medium for 2 weeks. (B) Germination rates of the transgenic and WT seedlings under normal conditions and oxidative treatment for $7 \mathrm{~d}$. (C) Phenotype of seedlings of each line after $14 \mathrm{~d}$. (D) Root length and (E) Fresh weight were obtained from seedlings that were transferred to MV-containing MS medium. (F) Phenotype of leaf discs after MV treatment. (G) Chlorophyll content in the leaf discs after MV treatments. The experiments were repeated three times and the bars indicate SEs. * and ** indicate significant differences at $\mathrm{P}<0.05$ and $\mathrm{P}<0.01$ in the values among the three overexpression lines and the WT plants.

of transgenic leaves was higher than WT under drought stress, which resulting in more $\mathrm{CO} 2$ accumulation inside the stroma of the chloroplast, however, the Ci (Fig. 3D)of the transgenic plants was lower than WT since their higher Pn (Fig. 3A). These results also suggest the improved drought stress tolerance by overexpression of TaPUB1.

Drought stress substantially decreased the water contents of the plant tissue, which had pronounced effects on photosynthetic rate and plant growth ${ }^{36}$. Our results showed that, compared to WT, the OE lines had lower water loss rate and higher RWC (Fig. 4A-C), this may be related to the lower osmotic potential (Fig. 4D) although the transpiration rate of the transgenic plants was higher (Fig. 3B) than the WT plants. Plants can accumulate compatible osmolytes to adjust the intracellular osmotic potentials in response to drought stress. Proline and soluble sugars, as compatible osmolytes, are thought to function mainly in osmotic adjustment and crucial to sustain cellular functions under drought stress ${ }^{5,37}$. The high water retention ability in the OE lines might partially be a result of the increase in the contents of compatible osmolytes (Fig. $4 \mathrm{E}$ and F).

Drought stress can inhibit the photosynthetic activity in tissues due to an imbalance between light capture and its utilization ${ }^{38}$. Down regulation of photosystem (PS) II activity results in changes in the quantum yield. These changes in the photochemistry of chloroplasts in the leaves of drought-stressed plants result in the dissipation of excess light energy in the PSII core and antenna, thus generating ROS, which are potentially dangerous under drought stress conditions ${ }^{39}$. ROS plays a crucial role in causing cellular damage under drought stress ${ }^{40}$. Injury caused by ROS to biological macromolecules under drought stress is among the major deterrents to plant growth ${ }^{5}$. The membranes of plant cells are the main targets of oxidative damage induced by drought stress, and cell membrane stability has been widely used for the estimation of stress tolerance ${ }^{41}$. The degree of injury to cell membrane induced by dehydration stress can be evaluated by detection of the electrolyte leakage from the leaves, and the intracellular MDA content is commonly used as an indicator of the degree of lipid peroxidation ${ }^{42,43}$. The transgenic plants maintained lower levels of electrolyte leakage and MDA content than did the WT plants after the drought treatment (Fig. 5A and B). ROS can attack most of the biological macromolecules, such as 
intracellular proteins, and damage their function ${ }^{40}$. There are less accumulation of protein carbonyl content in the transgenic plants (Fig. 5C and D) than that in WT. These results demonstrated that the TaPUB1 overexpression alleviated the oxidative damage in plants.

Furthermore, low levels of $\mathrm{H}_{2} \mathrm{O}_{2}$ and $\mathrm{O}_{2}^{--}$were detected in the $\mathrm{OE}$ plants under drought stress (Fig. 6). The low level of ROS in the OE plants was due to the higher activity of antioxidant enzymes, such as SOD, POD, CAT, and APX (Supplementary Fig. S1). The higher levels of proline and soluble sugars in the OE plants might also have participated in ROS scavenging ${ }^{37}$. These results suggest that the enhanced drought stress tolerance of OE plants maybe associate with the active oxygen scavenging system.

Drought can trigger a series of plant responses, including change in the level of gene expression ${ }^{44}$. These genes play a role in the stress response and function in stress tolerance at the molecular level ${ }^{4}$. Hence, we detected the expression of some of the antioxidant- and drought-related genes. As shown in Fig. 7, the expression levels of all these stress-inducible marker genes were increased by drought stress, and a larger increase was observed in the OE plants than in the WT plants. This suggest that the improved stress tolerance of OE plants might be related to the changed expression of stress-related genes, but the underlying mechanism is unclear.

Many stresses, such as salt and drought stress, disrupt the cellular homeostasis by enhancing the production of $\operatorname{ROS}^{45}$. Therefore, oxidative stress is a secondary stress involved in almost all the environment stresses ${ }^{46}$. The overexpression of TaPUB1 decreased the oxidative damage and enhanced the activity of antioxidant enzymes in the transgenic plants under drought stress conditions (Figs 5 and 6 and S1). Further, we detected the antioxidative compete of the transgenic lines in the present study, MV was used to induce oxidative stress. The OE plants had higher seed germination rates and longer roots than the WT plants under oxidative stress condition (Fig. 8A-E). At the same time, the leaf disc experiment also suggested a lower damage in the transgenic plants than that in the WT plants under oxidative stress (Fig. $8 \mathrm{~F}$ and G). The results shown in Supplementary Fig. S2 indicated the less ROS accumulation and higher antioxidant activity in the OE plants than that in the WT plants after MV treatment. Therefore, the overexpression of TaPUB1 improved the oxidative stress tolerance of the OE lines, which might be involved in their drought stress tolerance.

In conclusion, the data obtained in this study clearly suggest that overexpression of the U-box gene, TaPUB1, enhanced the drought tolerance of transgenic plants, probably by maintaining high water retention and strong antioxidant capacity. The antioxidant capacity might be one of the main mechanisms that enhanced the drought tolerance of the OE plants.

\section{Materials and Methods}

Plant materials and growth and stress conditions. The transgenic N. benthamiana plants containing the 35S::TaPUB1 vector (OE9, OE10, OE11) and the WT plants were used in this study. The seeds of $N$. benthamiana were sterilized with $70 \%$ alcohol for $2 \mathrm{~min}$, treated with $4 \%$ sodium hypochlorite solution for $8 \mathrm{~min}$, and then rinsed 5 times with sterilized water and grown, thereafter, in Petri dishes containing MS medium. The $N$. benthamiana seeds were sown in roseite in a growth room at $25^{\circ} \mathrm{C}$, under a $16-\mathrm{h} \mathrm{light} / 8$-h dark cycle with $200 \mu \mathrm{mol} \mathrm{m} \mathrm{m}^{-2} \mathrm{~s}^{-1}$ photon flux density. For the drought and MV treatments, 6-weeks-old seedlings were grown in solutions containing $10 \%$ PEG6000 (w/v) or $100 \mathrm{mM} \mathrm{MV}$, respectively. To analyze their drought tolerance, $N$. benthamiana plants were grown under normal water conditions for 6 weeks and then were deprived of irrigation completely for 5 or $7 \mathrm{~d}$.

Measurement of E3 ligase activity. $\quad 0.1 \mathrm{~g}$ fresh leaves were ground with PBS (PH 7.2-7.4). Then according to the Plant E3/UBPL ELISA kit (Shanghai Kenuodi Biological Technology Co. Ltd.) instructions, we add $50 \mu 1$ of sample to the sample port. Next add $100 \mu \mathrm{l}$ of enzyme conjugate to standard wells and sample wells except the blank well and incubate for $60 \mathrm{~min}$ at $37^{\circ} \mathrm{C}$. The following wash the Microtiter Plate 4 times. Then Add Substrate A $50 \mu \mathrm{l}$ and Substrate B $50 \mu \mathrm{l}$ to each well. After incubate for $15 \mathrm{~min}$ at $37^{\circ} \mathrm{C}$, add $50 \mu \mathrm{l} \mathrm{Stop} \mathrm{Solution}$. Last, read the Optical Density (O.D.) at $450 \mathrm{~nm}$ using a microtiter plate reader within $15 \mathrm{~min}$.

RNA extraction and cDNA synthesis. To analyze the response of overexpression-TaPUB1 plants to water stress, we extracted the total RNA from the two-month-old $N$. benthamiana leaves after drought treatment or normal water condition using Trizol reagent (TaKaRa, Japan) according to the operation instructions and performed the reverse transcription as described above. $2 \mu \mathrm{g}$ total RNA was subjected to synthesize cDNA using the HiFiScript Quick gDNA Removal cDNA Kit (Cwbiotech, China) according to the kit instructions. Then, the RT-qPCR was performed with a Bio-Rad CFX96TM real-time PCR system and TransStart Green qPCR SuperMix (TransGen Biotech, China). The PCR thermal cycle is programmed as follows reaction conditions: $94^{\circ} \mathrm{C}$ for $60 \mathrm{~s}$, 40 cycles at $94^{\circ} \mathrm{C}$ for $15 \mathrm{~s}, 56^{\circ} \mathrm{C}$ for $30 \mathrm{~s}$, then $72^{\circ} \mathrm{C}$ for $15 \mathrm{~s}$. The $N$. benthamiana $\beta$-actin gene was used as reference and the real-time PCR reaction primers are listed in Table 1.

Measurement of photosynthetic parameters and chlorophyll content. The photosynthetic parameters of adult tobacco lines were measured by a portable photosynthetic system (CIRAS-2 Hitchin, USA). The measurements were carried out under the condition of a $\mathrm{CO}_{2}$ concentration of $360 \mu \mathrm{l} \mathrm{l}^{-1}$, PFD of $800 \mu \mathrm{mol} \mathrm{m}^{-2}$ $\mathrm{s}^{-1}$, relative humidity of $60-70 \%$ and the temperature of the leaf chamber was $25^{\circ} \mathrm{C}$. First, all lines were lighted at least $30 \mathrm{~min}$ to induce the stomata open, then lighted at $800 \mu \mathrm{mol} \mathrm{m}^{-2} \mathrm{~s}^{-1} \mathrm{PDF}$ for $15 \mathrm{~min}$ to be acclimated.

About $0.1 \mathrm{~g}$ fresh leaves of each line were placed in $95 \%$ alcohol, and then extraction $40 \mathrm{~h}$ in darkness. The quantification was examined with a spectrophotometer. The chlorophyll content was calculated according to the method described of Kim and $\mathrm{Kim}^{47}$.

Measurements of leaf water loss, relative water content, osmotic potential, proline content, and soluble sugar contents. The two-month adult WT and OE lines without water in a greenhouse for 5 


\begin{tabular}{|l|l|}
\hline \multicolumn{2}{|l|}{ Sequence of the primers used in PCR analysis } \\
\hline \multicolumn{2}{|l|}{ The cloning of full-length cDNA } \\
\hline TaPUB1-F & ATGATCTGCGCGATCTCCGG \\
\hline TaPUB1-R & \multicolumn{1}{l|}{ TTGCGCCGCTGACTCTGATTTTC } \\
\hline Sequence of the primers used in qRT-PCR analysis \\
\hline q-TaPUB1-F & AAATCTCCAGTCATCCACTTCAC \\
\hline q-TaPUB1-R & CCATCTTCATTACCTTGCCATAC \\
\hline Nb3-actin-F & TGGACTCTGGTGATGGTGTC \\
\hline Nb3-actin- $\mathrm{R}$ & CCTCCAATCCAAACACTGTA \\
\hline NbCAT-F & GACATCACCTTACCT \\
\hline NbCAT-R & GAAGTTGTTCCCTACCAGAT \\
\hline NbGST-F & ACCCTTACCTTTCCCTCA \\
\hline NbGST-R & TTCCTTCACAGCAGCATC \\
\hline NbAPX-F & GGATTGGTTGCTGTTGAA \\
\hline NbAPX-R & CTTGAGGTAGGAGTTGTCG \\
\hline NbSOD-F & CAACTCCACGGCTTCCAGAC \\
\hline NbSOD-R & TGGGTCCTGATTAGCAGTGGT \\
\hline NbLEA-F & CCTTACTCTGTTCCTATTCC \\
\hline NbLEA-R & TTCTTCCTGATGCTATTACC \\
\hline NbRbohA-F & ACACACGCCATCAGAACTCCA \\
\hline NbRbohA-R & CCCACCCAACCAAAATACGC \\
\hline NbDREB-F & GAATAACCCCAAGAGGCG \\
\hline NbDREB-R & AGTCAGCGAAGTTCAAGCAA \\
\hline NbERD-F & CACTGATAAGAACTATGCGTTCAC \\
\hline NbERD-R & CTAAGCTAATCACATTCAGCGAG \\
\hline NbNCED-F & CGACCCACGAGTCCAGATTTC \\
\hline NbNCED-R & GAGCCTAGCAATTCCCGAGTG \\
\hline NbP5CS-F & AGAGGTGATGGAAGATTAGC \\
\hline NbP5CS-R & CCAACTGACCGAATAACG \\
\hline
\end{tabular}

Table 1. Primers used in this study.

days. The leaf water loss rate kinetics was implemented refer to $\mathrm{Li}$ et al. ${ }^{48}$. The osmotic potential of the leaves was assayed as described by Michel et al. ${ }^{49}$. The leaves relative water content were measured as described by González and González-Vilar ${ }^{50}$, the same the proline content and soluble sugar were measured according to the method of Hui et al. ${ }^{51}$. RWC computational formula: RWC $(\%)=(F W-D W) /(T W-D W) \times 100$. FW: fresh weight, TW: turgid weight, DW: dry weight.

MDA content and relative electrical conductivity. The MDA levels and relative electrical conductivity were measured based on description of Li et al. ${ }^{48}$.

Western blot analysis. Total protein was extracted from $N$. benthamiana leaves using protein extraction buffer ${ }^{52}$. The proteins separated by SDS-PAGE were electrotransfered to a PVDF membrane (Millipore, Billerica, MA) and then, incubated with 2,4-dinitrophenylhydrazine (DNPH, $0.1 \mathrm{mg} \mathrm{ml}^{-1}$ ) dissolved in $2 \mathrm{M} \mathrm{HCl}$. Thereafter, anti-DNP antibody was used to detect the level of protein carbonylation.

Histochemical ROS staining, measurements of $\mathrm{H}_{2} \mathrm{O}_{2}$ content and $\mathrm{O}_{2} \cdot-$ production rate. Hydrogen peroxide $\left(\mathrm{H}_{2} \mathrm{O}_{2}\right)$ and superoxide $\left(\mathrm{O}_{2}^{--}\right)$accumulation were dected with the staining methods described by Lu et al. ${ }^{53}$. Quantitative measurements of $\mathrm{H}_{2} \mathrm{O}_{2}$ and $\mathrm{O}_{2}^{--}$accumulation followed the methods described by Hui et al. ${ }^{51}$ and Wang et al. ${ }^{54}$.

Assay of antioxidant enzyme activity. The determination of SOD, CAT, POD and APX activities were measured as previously described ${ }^{55}$. The Shimadzu (UV-2550) spectrophotometer (Shimadzu, Tokyo, Japan) was used to spectrophotometric analyses.

Statistical Analysis. Each experiment included three repetition. The experimental data analysis was performed using the data processing system (Zhejiang University, China). Statistical significance was tested using Duncan's test at the 0.05 and 0.01 probability levels.

\section{References}

1. Niklas, K. J. Functional adaptation and phenotypic plasticity at the cellular and whole plant level. J Biosciences 34, 613-620 (2009).

2. Osakabe, Y., Osakabe, K., Shinozaki, K. \& Tran, L. S. P. Response of plants to water stress. Front Plant Sci 5, 86 (2014).

3. Smirnoff, N. The role of active oxygen in the response of plants to water deficit and desiccation. New Phytol 125, 27-58 (1993). 
4. Shinozaki, K. \& Yamaguchi-Shinozaki, K. Gene networks involved in drought stress response and tolerance. J Exp Bot 58, 221-227 (2007).

5. Farooq, M., Wahid, A. \& Kobayashi, N. et al. Plant drought stress: effects, mechanisms and management. Agron Sustain Dev 29, 185-212 (2009).

6. Sullivan, J. A., Shirasu, K. \& Deng, X. W. The diverse roles of ubiquitin and the $26 \mathrm{~S}$ proteasome in the life of plants. Nat Rev Genet 4, 948-958 (2003).

7. Dreher, K. \& Callis, J. Ubiquitin, hormones and biotic stress in plants. Ann Bot-London 99, 787-822 (2007).

8. Vierstra, R. D. The ubiquitin-26S proteasome system at the nexus of plant biology. Nat Rev Mol Cell Biol 10, 385-397 (2009).

9. Sadanandom, A., Bailey, M. \& Ewan, R. et al. The ubiquitin-proteasome system: central modifier of plant signalling. New Phytol 196, $13-28(2012)$

10. Smalle, J. \& Vierstra, R. D. The ubiquitin 26 S proteasome proteolytic pathway. Annu. Rev. Plant Biol. 55, 555-590 (2004).

11. Azevedo, C., Santos-Rosa, M. J. \& Shirasu, K. The U-box protein family in plants. Trends Plant Sci 6, 354-358 (2001).

12. Hatakeyama, S., Yada, M. \& Matsumoto, M. et al. U box proteins as a new family of ubiquitin-protein ligases. J Biol Chem 276, $33111-33120$ (2001).

13. Hu, X., Qian, Q. \& Xu, T. et al. The U-box E3 ubiquitin ligase TUD1 functions with a heterotrimeric G $\alpha$ subunit to regulate brassinosteroid-mediated growth in rice. PLoS Genet 9, e1003391 (2013).

14. Liu, P., Sherman-Broyles, S. \& Nasrallah, M. E. et al. A cryptic modifier causing transient self-incompatibility in Arabidopsis thaliana. Curr Biol 17, 734-740 (2007).

15. González-Lamothe, R., Tsitsigiannis, D. I. \& Ludwig, A. A. et al. The U-box protein CMPG1 is required for efficient activation of defense mechanisms triggered by multiple resistance genes in tobacco and tomato. Plant Cell 18, 1067-1083 (2006).

16. Monaghan, J., Xu, F. \& Gao, M. et al. Two Prp19-like U-box proteins in the MOS4-associated complex play redundant roles in plant innate immunity. PLoS Pathog 5, e1000526 (2009).

17. Seo, D. H., Ryu, M. Y. \& Jammes, F. et al. Roles of four Arabidopsis U-box E3 ubiquitin ligases in negative regulation of abscisic acidmediated drought stress responses. Plant Physiol 160, 556-568 (2012).

18. Wang, N., Liu, Y. \& Cong, Y. et al. Genome-Wide Identification of Soybean U-Box E3 Ubiquitin Ligases and Roles of GmPUB8 in Negative Regulation of Drought Stress Response in Arabidopsis. Plant Cell Physiol 57, 1189-1209 (2016).

19. Shao, H. B., Chu, L. Y. \& Wu, G. et al. Changes of some anti-oxidative physiological indices under soil water deficits among 10 wheat (Triticum aestivum L.) genotypes at tillering stage. Colloid Surface B 54, 143-149 (2007).

20. Asseng, S., Foster, I. A. N. \& Turner, N. C. The impact of temperature variability on wheat yields. Global Change Biol 17, 997-1012 (2011).

21. Oliver, C. N. Inactivation of enzymes and oxidative modification of proteins by stimulated neutrophils. Arch Biochem Biophys 253, 62-72 (1987)

22. Dudek, E. J., Shang, F. \& Valverde, P. et al. Selectivity of the ubiquitin pathway for oxidatively modified proteins: relevance to protein precipitation diseases. Faseb J 19, 1707-1709 (2005).

23. Foyer, C. H. \& Noctor, G. Redox homeostasis and antioxidant signaling: a metabolic interface between stress perception and physiological responses. Plant Cell 17, 1866-1875 (2005).

24. Dat, J., Vandenabeele, S. \& Vranová, E. et al. Dual action of the active oxygen species during plant stress responses. Mol Life S 57, 779-795 (2000).

25. Hatakeyama, S. \& Kei-ichi, I. N. U-box proteins as a new family of ubiquitin ligases. Biochem Bioph Res Co 302, 635-645 (2003).

26. Liu, J., Li, W. \& Ning, Y. et al. The U-box E3 ligase SPL11/PUB13 is a convergence point of defense and flowering signaling in plants. Plant Physiol 160, 28-37 (2012).

27. Luo, Q., Li, Y. \& Wang, W. et al. Genome-wide survey and expression analysis of Chlamydomonas reinhardtii U-box E3 ubiquitin ligases (CrPUBs) reveal a functional lipid metabolism module. PLoS One 10, e0122600 (2015).

28. Wang, C., Duan, W. \& Riquicho, A. R. M. et al. Genome-wide survey and expression analysis of the PUB family in Chinese cabbage (Brassica rapa ssp. pekinesis). Mol Genet Genomics 290, 2241-2260 (2015).

29. Zeng, L. R., Park, C. H. \& Venu, R. C. et al. Classification, expression pattern, and E3 ligase activity assay of rice U-box-containing proteins. Mol Plant 1, 800-815 (2008).

30. Yan, J., Wang, J. \& Li, Q. et al. AtCHIP, a U-box-containing E3 ubiquitin ligase, plays a critical role in temperature stress tolerance in Arabidopsis. Plant Physiol 132, 861-869 (2003).

31. Park, J. J., Yi, J. \& Yoon, J. et al. OsPUB15, an E3 ubiquitin ligase, functions to reduce cellular oxidative stress during seedling establishment. Plant J 65, 194-205 (2011).

32. Jian, H., Wang, J. \& Wang, T. et al. Identification of Rapeseed MicroRNAs Involved in Early Stage Seed Germination under Salt and Drought Stresses. Front Plant Sci 7, 658 (2016).

33. Rajjou, L., Duval, M. \& Gallardo, K. et al. Seed germination and vigor. Annu Rev Plant Biol 63, 507-533 (2012).

34. Flexas J, Bota J, Loreto F, et al. Diffusive and metabolic limitations to photosynthesis under drought and salinity in C3 plants. Plant Biol 6, 269-279 (2004).

35. Pinheiro, C. \& Chaves, M. M. Photosynthesis and drought: can we make metabolic connections from available data? J Exp Bot 62, 869-882 (2011)

36. Siddique, M. R. B., Hamid, A. \& Islam, M. S. Drought stress effects on water relations of wheat. Bot. Bull. Acad. Sinica 41, 35-39 (2001).

37. Xiong, L. \& Zhu, J. K. Molecular and genetic aspects of plant responses to osmotic stress. Plant Cell Environ 25, 131-139 (2002).

38. Foryer, C. H. \& Noctor, G. Oxygen processing in photosynthesis: regulation and signaling. New Phytol 146, 359-388 (2000).

39. Peltzer, D., Dreyer, E. \& Polle, A. Differential temperature dependencies of antioxidative enzymes in two contrasting species: Fagus sylvatica and Coleus blumei. Plant Physiol Bioch 40, 141-150 (2002).

40. Reddy, A. R., Chaitanya, K. V. \& Vivekanandan, M. Drought-induced responses of photosynthesis and antioxidant metabolism in higher plants. J Plant Physiol 161, 1189-1202 (2004).

41. Yue, Y., Zhang, M. \& Zhang, J. et al. Arabidopsis LOS5/ABA3 overexpression in transgenic tobacco (Nicotiana tabacum cv. Xanthi$n c)$ results in enhanced drought tolerance. Plant Sci 181, 405-411 (2011).

42. Bajji, M., Kinet, J. M. \& Lutts, S. The use of the electrolyte leakage method for assessing cell membrane stability as a water stress tolerance test in durum wheat. Plant Growth Regul 36, 61-70 (2002).

43. Sun, L., Liu, Y. \& Kong, X. et al. ZmHSP16. 9, a cytosolic class I small heat shock protein in maize (Zea mays), confers heat tolerance in transgenic tobacco. Plant Cell Rep 31, 1473-1484 (2012).

44. Umezawa, T., Fujita, M. \& Fujita, Y. et al. Engineering drought tolerance in plants: discovering and tailoring genes to unlock the future. Curr Opin Biotech 17, 113-122 (2006).

45. Mittler, R. Oxidative stress, antioxidants and stress tolerance. Trends Plant Sci 7, 405-410 (2002).

46. Dinakar, C., Djilianov, D. \& Bartels, D. Photosynthesis in desiccation tolerant plants: energy metabolism and antioxidative stress defense. Plant Sci 182, 29-41 (2012).

47. Kim, J. H. \& Kim, W. T. The Arabidopsis RING E3 ubiquitin ligase AtAIRP3/LOG2 participates in positive regulation of high-salt and drought stress responses. Plant Physiol 162, 1733-1749 (2013).

48. Li, A. X., Han, Y. Y. \& Wang, X. et al. Root-specific expression of wheat expansin gene TaEXPB23 enhances root growth and water stress tolerance in tobacco. Environ Exp Bot. 110, 73-84 (2014). 
49. Michel, B. E. \& Kaufmann, M. R. The osmotic potential of polyethylene glycol 6000. Plant Physiol 51, 914-916 (1973).

50. González, L., González-Vilar, M. Determination of relative water content[M]//Handbook of plant ecophysiology techniques. Springer Netherlands 2001, 207-212.

51. Hui, Z., Tian, F. X. \& Wang, G. et al. The antioxidative defense system is involved in the delayed senescence in a wheat mutant tasg1. Plant Cell Rep 31, 1073-1084 (2012).

52. Lee, D. G., Ahsan, N. \& Lee, S. H. et al. An approach to identify cold-induced low-abundant proteins in rice leaf. C. R. Biol. 330, 215-225 (2007).

53. Lu, W., Chu, X. \& Li, Y. et al. Cotton GhMKK1 induces the tolerance of salt and drought stress, and mediates defence responses to pathogen infection in transgenic Nicotiana benthamiana. PLoS One 8, e68503 (2013).

54. Wang, C. F., Huang, L. L. \& Buchenauer, H. et al. Histochemical studies on the accumulation of reactive oxygen species $\left(\mathrm{O}_{2}{ }^{--}\right.$and $\mathrm{H}_{2} \mathrm{O}_{2}$ ) in the incompatible and compatible interaction of wheat-Puccinia striiformis f. sp. tritici. Physiol. Mol Plant P 71, 230-239 (2007).

55. Zhang, J., Guo, Q. F. \& Feng, Y. N. et al. Manipulation of monoubiquitin improves salt tolerance in transgenic tobacco. Plant Biology 14, 315-324 (2012).

\section{Acknowledgements}

This study was supported by the National Natural Science Foundation of China (No. 31370304) and by Funds of Shandong "Double Tops" Program.

\section{Author Contributions}

G.Z. and W.W. conceived the study. G.Z. performed most of the experiments. M.Z., Z.Z., Y.R. and Q.L. performed part of the experiments and took part in the discussion of project. G.Z. wrote the manuscript.

\section{Additional Information}

Supplementary information accompanies this paper at doi:10.1038/s41598-017-08181-w

Competing Interests: The authors declare that they have no competing interests.

Publisher's note: Springer Nature remains neutral with regard to jurisdictional claims in published maps and institutional affiliations.

(c) (i) Open Access This article is licensed under a Creative Commons Attribution 4.0 International

License, which permits use, sharing, adaptation, distribution and reproduction in any medium or format, as long as you give appropriate credit to the original author(s) and the source, provide a link to the Creative Commons license, and indicate if changes were made. The images or other third party material in this article are included in the article's Creative Commons license, unless indicated otherwise in a credit line to the material. If material is not included in the article's Creative Commons license and your intended use is not permitted by statutory regulation or exceeds the permitted use, you will need to obtain permission directly from the copyright holder. To view a copy of this license, visit http://creativecommons.org/licenses/by/4.0/.

(c) The Author(s) 2017 